\title{
Quantification of the Ozone Dose Delivered into a Liquid by Indirect Plasma Treatments: Method and Calibration of the Pittsburgh Green Fluorescence Probe
}

\author{
A. Wright ${ }^{1,3} \cdot$ J. Fuster ${ }^{2} \cdot$ A. Shaw ${ }^{3} \cdot$ H. Bandulasena ${ }^{1} \cdot$ B. R. Buckley ${ }^{2} \cdot$ F. Iza ${ }^{3}$
}

Received: 30 April 2018 / Accepted: 31 July 2018 / Published online: 21 August 2018

(c) The Author(s) 2018

\begin{abstract}
Determination of the ozone dose delivered into liquids by plasma systems is of importance in many emerging plasma applications, such as plasma medicine. Quantification of this dose remains extremely challenging due to the complex physico-chemical processes encountered in the gas plasma, the plasma-liquid interface and the liquid itself. Chemical probes have the potential to address the limitation of more traditional plasma diagnostic techniques but most commercial chemical probes are not specific enough to be used in plasma applications. Here we report on the development of a method for the quantification of the ozone delivered into a liquid using Pittsburgh Green, a novel ozone-selective fluorescence probe. Entailed within this work is a method for the preparation of the probe solutions, the design of a calibration system and a normalized calibration curve correlating fluorescence intensity to actual ozone dose delivered to the liquid. This enables the quantitative comparison of ozone measurements performed with different spectrofluorometers and in different institutions.
\end{abstract}

Keywords Plasma-liquid $\cdot$ Ozone $\cdot$ Fluorescent probe $\cdot$ Pittsburgh green

\section{Introduction}

Ozone $\left(\mathrm{O}_{3}\right)$, traditionally used for drinking and waste water treatment $[1,2]$, has received increasing attention in recent years due to its importance in atmospheric science [3] and its potential use in emerging plasma applications such as food sanitization [4], plasma medicine and surface cleaning [5,6]. The predominant source of ozone for

B. R. Buckley

b.r.buckley@lboro.ac.uk

$\triangle$ F. Iza

f.iza@lboro.ac.uk

1 Department of Chemical Engineering, Loughborough University, Loughborough LE11 3TU, UK

2 Department of Chemistry, Loughborough University, Loughborough LE11 3TU, UK

3 Wolfson School of Mechanical, Electrical and Manufacturing Engineering, Loughborough University, Loughborough LE11 3TU, UK 
these applications is atmospheric-pressure plasma, where ozone is produced alongside a broad range of other reactive oxygen and nitrogen species. In many cases, ozone is delivered to a liquid phase, and to advance our understanding and to optimize these systems, it is necessary not only to measure the concentration of ozone in the gas phase but also to quantify the amount of ozone that is actually delivered to the liquid.

Quantitative measurement of the ozone dose in these systems is challenging, as it requires a high degree of selectivity. In the gas phase, ozone is often measured using Fourier transform infrared (FTIR) spectroscopy [7]. Although well-developed for the detection of ozone in gas phase, this method is not feasible for the measurement of ozone in liquids as infrared transparent windows are water-soluble. An alternative technique to measure ozone is ultra-violet (UV) absorption in the Hartley band (220-290 nm). This can be used in both gas and liquid phases as long as UV transparent windows, such as quartz, are used in the measurements $[8,9]$. However, water and many organic compounds, such as those encountered in cell culture media, absorb in the UV, limiting the applicability of UV absorption in many practical scenarios [10, 11].

An alternative method for measuring the dose of ozone delivered to a liquid by a plasma treatment is the use of chemical probes. One of the most commonly used probes is indigo carmine (indigo disulfonate), a water soluble blue dye that in the presence of ozone readily undergoes ozonolysis. This causes the eventual cleavage of its central $\mathrm{C}=\mathrm{C}$ double bond, as indicated in Fig. 1 [12]. This reaction can be monitored optically as indigo absorbs light at $605 \mathrm{~nm}$ and the product of the ozonolysis does not, making it convenient and easy to use.

However, indigo does not react selectively with ozone, and many other reactive oxygen species often present in plasmas such as hydroxyl radicals and singlet oxygen can cause the cleavage of the double bond $[13,14]$. As a result, indigo is unsuitable as a diagnostic technique for ozone in applications where other oxidative species may be present. Unfortunately, this is the case of most plasma treatments. Another chemical technique often reported in the literature to measure ozone employs KI. In the presence of ozone, KI forms iodine $\left(\mathrm{I}_{2}\right)$, and the concentration of iodine can be determined via titration [15]. There are still open questions over the robustness of this method as results are very sensitive to a range of parameters; such as buffer concentration/composition, $\mathrm{pH}$ and sampling techniques. KI solutions are also known to react with other reactive oxygen species such as hydroxyl radicals [16].

Recently, a chemical probe that is sensitive and specific to ozone, Pittsburgh green (PG), has been reported [17]. In the presence of ozone, PG reacts to form a fluorescent product (see Fig. 2) that can be detected optically. Ozone reacts with PG (1) via a 1,3-dipolar cycloaddition, producing a molozonide (2) before a further step of a retro$[3+2]$ cyclization forms an aldehyde (3). The final step, a $\beta$-elimination of the aldehyde to form the fluorescent product (4) is the rate limiting step and can take in the order of

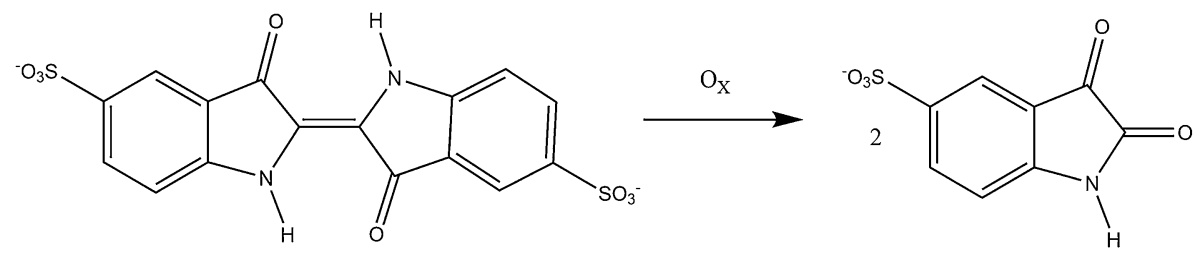

Fig. 1 Oxidation reaction of indigo disulfonate 


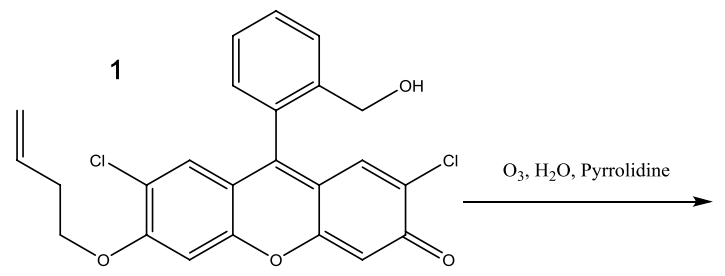<smiles>O=c1cc2oc3cc(O)c(Cl)cc3c(-c3ccccc3CO)c-2cc1Cl</smiles>

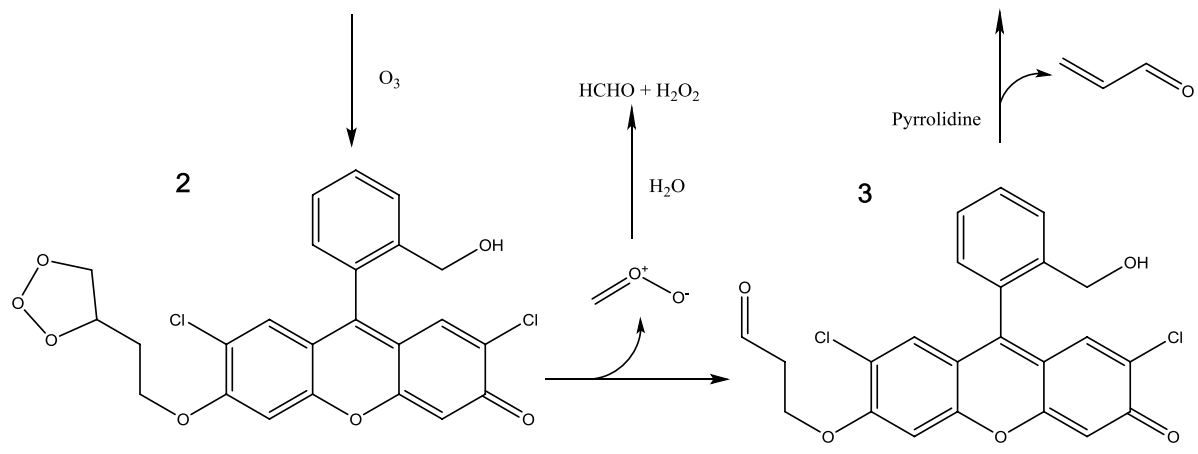

Fig. 2 The reaction scheme of Pittsburgh green (PG) to form its fluorescent product after reacting with $\mathrm{O}_{3}$

hours to complete, but this can be catalysed in the presence of a base such as pyrrolidine [18].

Castello-Beltran et al. [19] studied the pH stability of PG and also reported that overexposure of the probe to ozone results in conversion of the fluorescent product (4) to $2^{\prime}, 7^{\prime}$-dichloro-fluorescein (DCF), affecting the quantification capability of the probe. Therefore, PG assays must be carried out with the probe in excess and preferably in a neutral $\mathrm{pH}$ buffer solution [20,21].

Despite its potential in terms of sensitivity and selectivity, PG is not commercially available and hence it has not been previously studied for its use in the detection of ozone in plasma-liquid systems. In this manuscript, we explore the use of PG for the quantification of ozone in liquid in a remote plasma system, developing a protocol for the quantification of the ozone dose delivered in remote plasma-liquid systems in concentrations up to hundreds of $\mu \mathrm{M}$, concentrations 3 orders of magnitude larger than in previous reports. A calibration curve is obtained and we present a method that can be used by researchers in other institutions to report quantitative results.

\section{Experimental Setup and Methods}

\section{The Fluorescent Probe Pittsburgh Green (PG)}

Although PG is currently not commercially available, its synthesis is relatively simple and high yielding. The probe is derived from $2^{\prime}, 7^{\prime}$-dichlorofluorescein and details of its synthesis can be found in [18]. The probe is specific over other reactive oxygen species often encountered in plasma systems such as singlet oxygen, hydroxyl radical and hydrogen peroxide [17], as well as reactive nitrogen species such as nitric oxide and peroxynitrite [19]. This makes the probe particularly interesting for plasma applications. 
As PG is not fully soluble in water, it is necessary to develop a solvent solution for the probe. In previous studies, PG has been dissolved in 5\% methanol in aqueous buffer solution [17]. The use of methanol, however, has some limitations: for biological applications, methanol is toxic to cells [22], and when used in plasma systems, methanol evaporates quickly due to its high volatility (boiling point $64.7^{\circ} \mathrm{C}$ ).

Therefore, to extend the use of PG to a plasma system, an alternative formulation for the solvent is required. Here we propose the use of dimethyl sulfoxide (DMSO) as DMSO has a higher boiling point $\left(189^{\circ} \mathrm{C}\right)$ and low toxicological impact [22].

To minimize changes to ozone solubility and $\mathrm{pH}$ over time, the PG solution used in the study was buffered. Probe solutions were prepared to a concentration of $300 \mu \mathrm{M}$ in 5\% DMSO in a phosphate $7.4 \mathrm{pH}$ buffer $(0.1 \mathrm{M})$ solution. All chemicals were sourced from Sigma, UK, except for the probe, which was synthesized in-house [19].

\section{Experimental Set Up}

Calibration of fluorescence probes is typically performed by preparing solutions of the fluorescent product in varying known concentrations and measuring the resulting fluorescence. The resulting calibration curve is specific for that particular probe and spectrometer system. This approach, however, could not be applied in this study as it would only provide quantification of the fluorescent product generated in the system but not of the ozone delivered to the solution. As it is discussed below, these two quantities are not necessarily the same.

Therefore, an alternative calibration system was developed to correlate the concentration of ozone delivered to the PG solution and the fluorescence of the solution. A schematic of the system is shown in Fig. 3. Probe solution $(25 \mathrm{ml})$ was placed in a Dreschel bottle and ozone produced in the afterglow of an atmospheric-pressure $\mathrm{N}_{2} / \mathrm{O}_{2}$ plasma was bubbled through the solution. The amount of ozone delivered to the Dreschel bottle and the ozone leaving through its exhaust were measured using UV absorption spectroscopy. Comparison of the amount of ozone delivered and leaving the Dreschel bottle enables accurate quantification of the amount of ozone trapped in the solution.

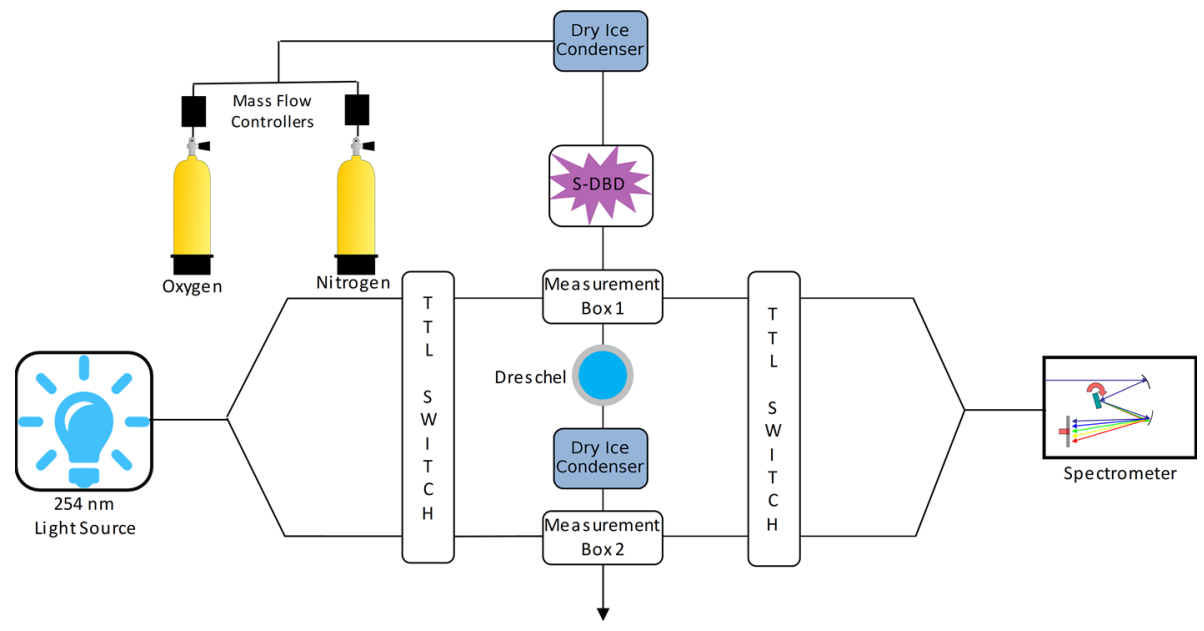

Fig. 3 A schematic of the setup used for the calibration of the ozone probe 
A gas mixture of $\mathrm{N}_{2}(90 \%)$ and $\mathrm{O}_{2}(10 \%)$ was used in the study with a gas flow rate of 0.5 standard litres per minute (SLPM) controlled by an Alicat MC-2SLPM mass flow controller. Similar results have been obtained with a gas mixture of $\mathrm{Ar}(90 \%)$ and $\mathrm{O}_{2}(10 \%)$, which in agreement with previous studies suggest that PG is not influenced by the presence of reactive nitrogen species. This is not the case for indigo solutions as even in the mild conditions used in this study the amount of indigo degraded is larger than the amount of ozone lost in the system due to reactions of indigo with other reactive species.

The gas was first passed through a dry ice condenser to remove any humidity in the gas supply before it entered a plasma chamber similar to that employed by Shaw et al. [23] A surface dielectric barrier discharge (s-DBD) driven by a modulated in-house built fullbridge resonant power supply was used to generate ozone. The s-DBD operated a 5\% duty cycle at $35 \mathrm{kHz}$ and $5.5 \mathrm{kV}_{\mathrm{pp}}$. The effluent of the s-DBD was then passed through the first of two ozone measurement boxes. The boxes are made of anodized aluminium and contain an inlet and outlet for the gas, with two quartz windows and collimated lenses in opposite sides of the box to provide optical access for UV absorption measurements. The gas was then directed into the Dreschel bottle where it was bubbled through and ozone was delivered to the solution. At the exit of the Dreschel bottle, a second dry ice condenser and a chilled water reflux condenser were used to remove humidity picked up by the gas in the Dreschel bottle. Finally, the gas was directed through a second ozone measuring box before it was released to the atmosphere through an ozone scrubber.

To measure the ozone concentration in the measuring boxes, a $255 \mathrm{~nm}$ LED light (Ocean optics, LLS-255) was directed into a bifurcated fibre (Ocean Optics, QP600-1XSR) to create two channels, one for each box (see Fig. 3). Two TTL switches (Ocean Optics, FOR-2X2-TTL) were used to control the light directed to the spectrometer (Ocean Optics, HR2000+). This arrangement requires only one UV source and one spectrometer, minimizing mismatches between the readings in each box. It can be shown that the ozone concentration difference between the two boxes $\left(\Delta\left[O_{3}\right]\right)$ in molecules $/ \mathrm{cm}^{3}$ between the two boxes is given by:

$$
\Delta\left[O_{3}\right]=\left[O_{3}\right]_{b o x 1}-\left[O_{3}\right]_{b o x 2}=A+\frac{1}{\sigma L} \ln \left(\frac{I_{2}}{I_{1}}\right)
$$

where $I_{1}$ and $I_{2}$ are the light intensities recorded through channel 1 and channel 2, respectively; $\sigma$ the cross section area at $255 \mathrm{~nm}\left(1.1 \times 10^{17} \mathrm{~cm}^{2}\right)$ [24]; $L$ the optical path length of the boxes $(7 \mathrm{~cm})$ and $A$ a constant that depends on the way light is split in the bifurcation fibres and any difference in attenuation within the fibres. Although difficult to predict theoretically, $A$ can be easily determined experimentally by measuring the light intensity in each channel when no ozone is present in the boxes (after a nitrogen purge) since the difference in ozone concentration in this case must be zero. The difference in ozone concentration $\left(\Delta\left[\mathrm{O}_{3}\right]\right)$ measured in this way is insensitive to drifts in the light source intensity or the spectrometer's sensitivity and can therefore be reliably used for measuring difference in ozone concentration over extended periods of time.

\section{Ozone Dose Measurement}

To obtain a calibration curve that relates fluorescence to ozone concentration, it is necessary to quantify the actual amount of ozone delivered to the liquid. This is achieved using the setup described in "Experimental Set Up" Section by measuring the difference in ozone concentration in the gas before and after the bubbling Dreschel bottle. 
An example of the time evolution of the difference in ozone concentration between box one and box two during an experiment is shown in Fig. 4a. Initially the system is flushed with the $\mathrm{N}_{2} / \mathrm{O}_{2}$ gas mixture for $15 \mathrm{~min}$ and during this time, the ozone difference between the two boxes is zero (see Fig. 4a). After $15 \mathrm{~min}$, the plasma is switched on and ozone starts to be produced. As ozone is transported through the system, ozone reaches box 1 before any ozone makes it to box 2 . This causes the rapid increase in concentration difference seen in Fig. 4a at 15 min. As ozone starts to reach box 2, the difference in ozone concentration between the two boxes decreases but remains positive throughout the experiment (15-40 min) as some ozone is lost in the system. When the plasma is switched off at 40 min, the first box fills with $\mathrm{N}_{2} / \mathrm{O}_{2}$ while box two continues to have some ozone. This causes the difference to become negative for a little while until the system is completely flushed out and the difference returns to zero.

By integrating the ozone difference and taking into account that the gas flow rate is 0.5 SLPM, it is possible to determine the amount of ozone that reacted in the system. Knowing the volume of the liquid in the Dreschel bottle $(25 \mathrm{ml})$, the amount of ozone can be converted to an equivalent ozone concentration in the liquid. This is shown in Fig. $4 \mathrm{~b}$.

The ozone lost in the system is primarily due to the reaction of ozone with the probe solution. Nonetheless, some ozone is lost in the system even if no liquid is present in the Dreschel bottle. This loss is attributed primarily to wall reactions in the system and it was found to be linear with respect to the duration of the experiment. Therefore, for each experiment, the amount of ozone delivered to the liquid was computed by subtracting the amount of ozone lost in the system when probe is not present from the total amount of ozone lost in the system with the probe in it.

The ozone dose delivered to the solution reacts with both the probe and DMSO. Therefore the fluorescence measurements need to be calibrated to the net ozone dose to

(a)
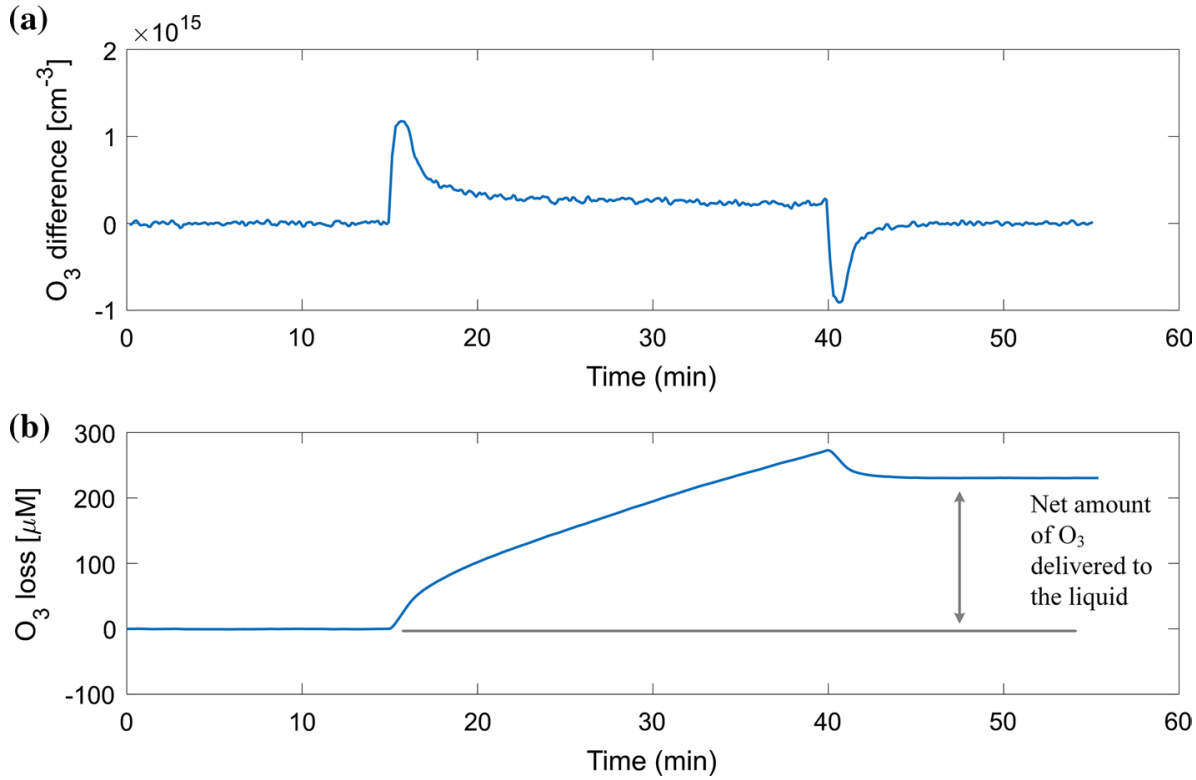

Fig. 4 a Time evolution of the $\mathrm{O}_{3}$ concentration difference between the first and second measuring box. b Net amount of $\mathrm{O}_{3}$ delivered to the liquid 
the liquid and not solely to the amount of ozone that reacts with the probe. As long as DMSO remains in excess (its concentration is more than 2000 times higher than that of the probe), reactions of DMSO with ozone or any other species such as $\mathrm{OH}$ [25], would not affect the validity of the results of this work. Similar considerations should be made if a different solvent is used.

\section{Fluorescence Measurement}

One of the shortcomings of PG is the slow $\beta$-elimination of the aldehyde (3) to form the fluorescent product (4), which can result in fluorescence readings varying over time [19]. To overcome this problem, in this study the $\beta$-elimination of the aldehyde was catalysed by the presence of pyrrolidine. After exposing a probe solution to ozone as described above, samples were prepared for fluorescence measurements by making up a solution of $5 \%$ treated probe to $95 \% \mathrm{pH} 9$ pyrrolidine solution $(47.5 \mathrm{mM}, 0.5 \mathrm{M}$ borate buffer) and left to stand for $3 \mathrm{~min}$ [18].

The absorbance and fluorescence spectra were measured with a UV-VIS spectrometer (Lambda 35, Perkin Elmer) to quantify the stoke shift. Figure 5 shows the absorption and fluorescence spectra of both an untreated solution of PG and a solution of PG after 70 min of ozone exposure. The stoke shift was found to be $\sim 20 \mathrm{~nm}$ and subsequent fluorescence measurements were performed with a spectrofluorometer (FluoroMax, Horiba Scientific) with an excitation wavelength of $496 \mathrm{~nm}$. Fluorescence values reported in this paper were measured by integrating the emission from 510 to $600 \mathrm{~nm}$.

It is worth noting in Fig. 5 that the PG exhibits a small amount of fluorescence before exposure to ozone, which as it will be shown later, can be used as a calibration reference to extend the use of the calibration curve produced in this study to experiments performed with different fluorescent spectrometers.

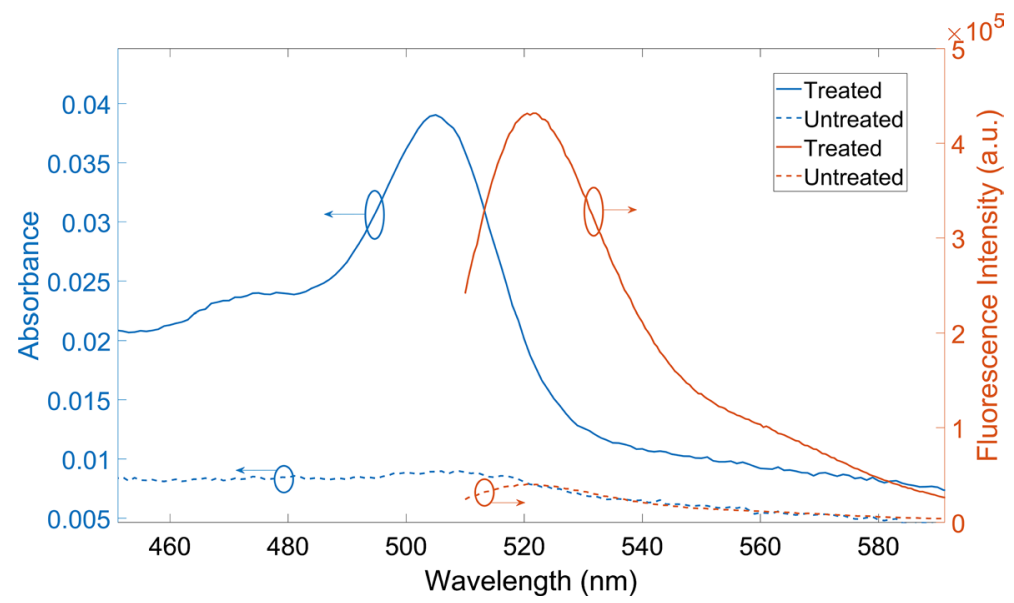

Fig. 5 Absorption and fluorescence spectra of Pittsburgh green (PG) before (untreated) and after 70 min of exposure to $\mathrm{O}_{3}$ (treated) (Color figure online) 


\section{Results and Discussion}

\section{Calibration Curve}

Probe solutions were treated in the calibration system for different time durations ranging from 0 to $145 \mathrm{~min}$. The ozone consumed in the experiment was determined as described above and the dose of ozone delivered to the liquid was then calculated by subtracting the background wall losses.

Figure 6 shows the correlation between the amount of ozone delivered to the liquid expressed in terms of concentration in a $25 \mathrm{ml}$ volume and the normalised fluorescence of the solution after the ozone exposure. The correlation is linear over a wide range of ozone concentrations and up to $\mu \mathrm{M}$ concentrations often encountered in plasma applications.

For the spectrometer used in this study, the initial fluorescence of PG before ozone exposure was 1,592,270 counts, which was normalized to a fluorescence intensity of 1 . It is important to note that although the probe solutions are $300 \mu \mathrm{M}$, DMSO also acts as an ozone scavenger [26], and therefore not all the ozone delivered to the liquid reacts with the probe. The same is likely to be true with other solution systems. Therefore, it is important to calibrate the fluorescence of the probe solution directly to the amount of ozone delivered to the liquid as it is done in this study instead of assuming that under experimental conditions there will be a 1:1 relationship between ozone and fluorescent molecules (even if this is the stoichiometry of the reaction). The calibration curve produced in this work (Fig. 6) is therefore valid as long as the probe solutions are prepared as described in "Experimental Setup and Methods" Section, and can be used to compare different plasma systems. Changing the solution composition would require a new calibration curve.

As a result of the competing reactions of ozone with the probe and DMSO, it is possible to detect ozone concentrations larger than the probe concentration in the solution. For example, in Fig. 6, a linear behaviour to ozone concentrations in excess of $500 \mu \mathrm{M}$ is observed even though the probe concentration is only $300 \mu \mathrm{M}$. Liquid chromatography mass spectrometry (LCMS) analysis of the samples exposed to the largest amount of ozone in Fig. 6 show still significance presence of unreacted probe.

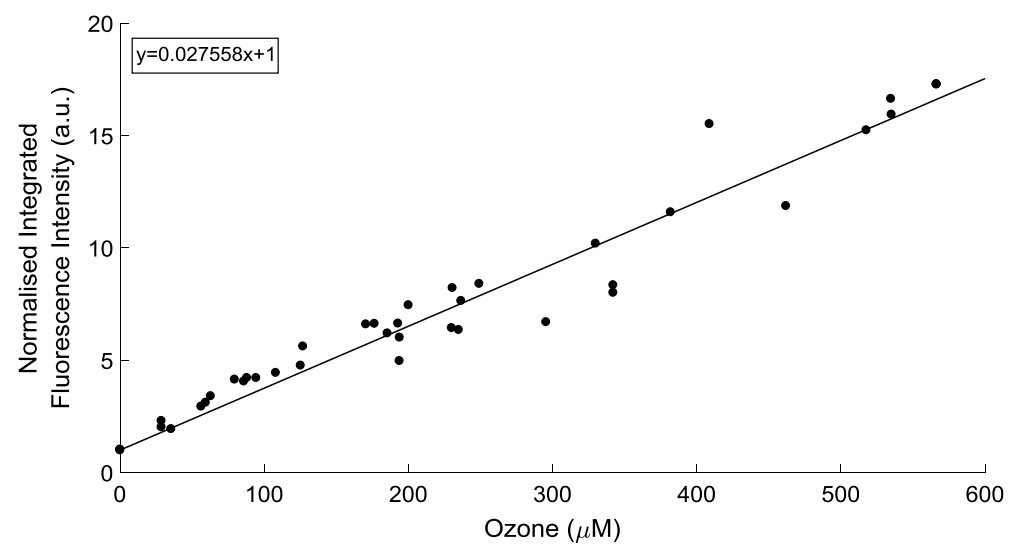

Fig. 6 Calibration plot of the Pittsburgh green (PG) probe 


\section{Calibration for Different Spectrometers}

One of the limitations of fluorescence methods is the difficulty to compare quantitatively fluorescence results obtained with different spectrometers. This is also the case for PG. However, as shown in Figs. 5 and 6, PG is slightly fluorescent even before it is exposed to ozone. While this may be a shortcoming when trying to measure small ozone concentrations $(<\mathrm{nM})$, this initial fluorescence can also be used to calibrate the fluorescence measurements obtained with different spectrometers.

To test the reliability of this approach, a separate batch of the PG probe solutions was treated in the same experimental set up so that the actual ozone dose could be quantitatively determined but with the fluorescence measured using a custom-built spectrometer set-up consisting of a cuvette holder (Ocean Optics, CUV-ALL-UV), collimating lenses and optical fibres (Ocean optics, QP600-1-XSR) placed at $90^{\circ}$, a spectrometer (Ocean Optics, HR2000+), a broad band LED light source (Ocean Optics, LS-475) and optical filter (Brightline, 536/40). With this system, the initial fluorescence intensity of PG before ozone exposure was 10,868 counts. The fluorescence of the PG as determined with this equipment $(10,868$ counts) is orders of magnitude different from the fluorescence measured with the FluoroMax spectrofluorometer (1,592,270 counts). Nonetheless, this value can be used to normalize the measurements performed with this set-up.

Figure 7 shows the normalized fluorescence data measured with the two optical systems as a function of the ozone dose delivered to the solution. A comparison of the two data sets shows a very good agreement, demonstrating that it is possible to use the self-fluorescence of the probe to normalize the measured fluorescence and use the calibration data provided in Fig. 6 to determine the ozone dose delivered to the liquid in other systems.

\section{Conclusion}

Chemical probes can provide a means to determine the dose of plasma generated species delivered to a liquid. To date, however, most probes have been developed for non-plasma environments. This implies that the interpretation of results obtained with these probes

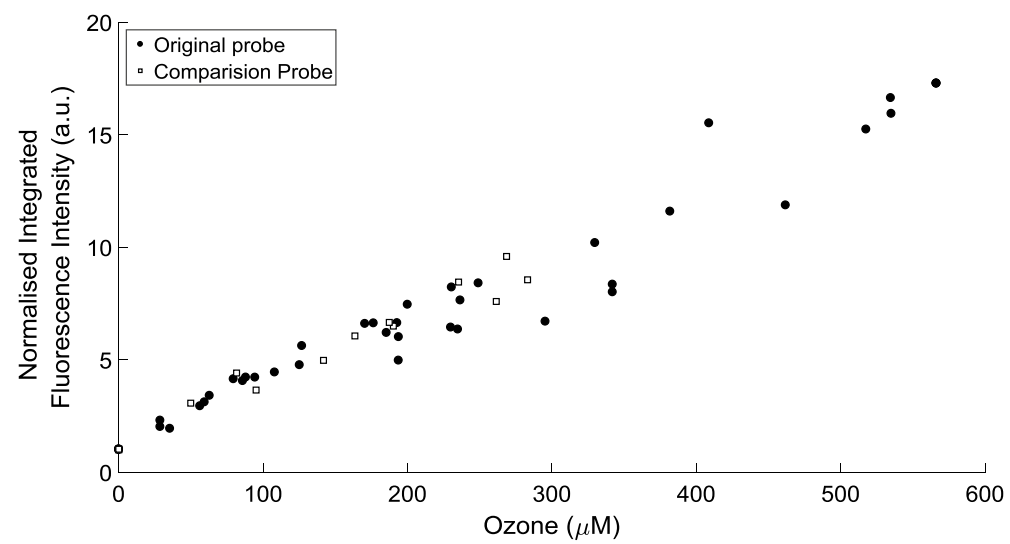

Fig. 7 A comparison of the normalized fluorescence (see text) measured with two different spectrometer systems 
needs to be carried out cautiously because the probes may not be selective under the reactive plasma environments.

Here we have demonstrated the use of Pittsburgh green (PG) as a selective probe for the determination of the dose of ozone delivered by a remote plasma system. Future studies should examine the suitability in direct plasma systems.

The composition of the probe solution is important as solvents can act as scavengers and lead to misinterpretation of fluorescence measurements. To circumvent this problem, an experimental setup was developed to correlate the fluorescence of the probe to the actual ozone dose delivered to the liquid. The correlation is linear up to ozone doses in the order of hundreds of $\mu \mathrm{M}$. A normalized calibration curve has been obtained and it was demonstrated that the small self-fluorescence of PG can be used to calibrate fluorescence measurements performed with different optical systems, allowing the quantitative application of this probe in other laboratories.

Acknowledgements The authors are grateful for the support of Loughborough University's Centre for doctoral training on Gas Plasma Interactions with Organic Liquids and thank Dr. Stephen Butler, Dr. Kristian Wende, Dr. Stephan Reuter and Dr. Helena Jablonowski, for helpful discussions. A.W. also acknowledges the support of UK Engineering and Physical Sciences Research Council (EPSRC).

Open Access This article is distributed under the terms of the Creative Commons Attribution 4.0 International License (http://creativecommons.org/licenses/by/4.0/), which permits unrestricted use, distribution, and reproduction in any medium, provided you give appropriate credit to the original author(s) and the source, provide a link to the Creative Commons license, and indicate if changes were made.

\section{References}

1. Von Gunten U (2003) Ozonation of drinking water: part I. Oxidation kinetics and product formation. Water Res 37:1443-1467. https://doi.org/10.1016/S0043-1354(02)00457-8

2. Jo J-O, Mok YS (2009) In-situ production of ozone and ultraviolet light using a barrier discharge reactor for wastewater treatment. J Zhejiang Univ Sci A 10:1359-1366. https://doi.org/10.1631/jzus.A0820 696

3. Monks PS, Archibald AT, Colette A et al (2015) Tropospheric ozone and its precursors from the urban to the global scale from air quality to short-lived climate forcer. Atmos Chem Phys 15:8889-8973. https://doi.org/10.5194/acp-15-8889-2015

4. Shaw A, Shama G, Iza F (2015) Emerging applications of low temperature gas plasmas in the food industry. Biointerphases 10:29402. https://doi.org/10.1116/1.4914029

5. Graves DB (2012) The emerging role of reactive oxygen and nitrogen species in redox biology and some implications for plasma applications to medicine and biology. J Phys D Appl Phys 45:263001263042. https://doi.org/10.1088/0022-3727/45/26/263001

6. Liu D-X, Rong M-Z, Wang X-H et al (2010) Main species and physicochemical processes in cold atmospheric-pressure $\mathrm{He}+\mathrm{O}_{2}$ plasmas. Plasma Process Polym 7:846-865. https://doi.org/10.1002/ ppap. 201000049

7. Bulanin KM, Lavalley JC, Tsyganenko AA (1995) IR spectra of adsorbed ozone. Colloids Surf A Physicochem Eng Asp 101:153-158. https://doi.org/10.1016/0927-7757(95)03130-6

8. Al-Abduly A, Christensen P (2015) An in situ and downstream study of non-thermal plasma chemistry in an air fed dielectric barrier discharge (DBD). Plasma Sources Sci Technol 24:65006. https://doi. org/10.1088/0963-0252/24/6/065006

9. van den Broeke J, Ross PS, van der Helm AWC et al (2008) Use of on-line UV/Vis-spectrometry in the measurement of dissolved ozone and AOC concentrations in drinking water treatment. Water Sci Technol 57:1169-1175. https://doi.org/10.2166/wst.2008.137

10. Rodríguez FJ, Schlenger P, García-Valverde M (2016) Monitoring changes in the structure and properties of humic substances following ozonation using UV-Vis, FTIR and ${ }^{1} \mathrm{H}$ NMR techniques. Sci Total Environ 541:623-637. https://doi.org/10.1016/j.scitotenv.2015.09.127 
11. Hofman-Caris RCHM, Harmsen DJH, Beerendonk EF et al (2012) Prediction of advanced oxidation performance in various pilot $\mathrm{UV} / \mathrm{H}_{2} \mathrm{O}_{2}$ reactor systems with MP- and LP- and DBD-UV lamps. Chem Eng J 210:520-528. https://doi.org/10.1016/j.cej.2012.09.041

12. Bader H, Hoigné J (1981) Determination of ozone in water by the indigo method. Water Res 15:449456. https://doi.org/10.1016/0043-1354(81)90054-3

13. Wentworth P (2002) Evidence for antibody-catalyzed ozone formation in bacterial killing and inflammation. Science 298(80):2195-2199. https://doi.org/10.1126/science.1077642

14. Kettle AJ, Clark BM, Winterbourn CC (2004) Superoxide converts indigo carmine to isatin sulfonic acid implications for the hypothesis that neutrophils produce ozone. J Biol Chem 279:18521-18525. https://doi.org/10.1074/jbc.M400334200

15. Lukes P, Clupek M, Babicky V et al (2005) Generation of ozone by pulsed corona discharge over water surface in hybrid gas-liquid electrical discharge reactor. J Phys D Appl Phys 38:409-416. https://doi. org/10.1088/0022-3727/38/3/010

16. Langlain B, Reckhow D, Brink D (1991) Ozone in water treatment: application and engineering. CRC Press, Boca Raton

17. Garner AL, Croix CMS, Pitt BR et al (2010) Specific fluorogenic probes for ozone in biological and atmospheric samples. Nat Chem 2:422. https://doi.org/10.1038/nchem.621

18. Leslie AK, Li D, Koide K (2011) Amine-promoted $\beta$-elimination of a $\beta$-aryloxy aldehyde for fluorogenic chemodosimeters. J Org Chem 76:6860-6865. https://doi.org/10.1021/jo200947e

19. Castelló BC, Palmer EA, Buckley BR, Iza F (2014) Virtues and limitations of Pittsburgh green for ozone detection. Chem Commun 51:1579-1582. https://doi.org/10.1039/c4cc08244c

20. Basu U, Koide K (2016) Comment on "virtues and limitations of Pittsburgh green for ozone detection" by C. C. Beltrán, E. A. Palmer, B. R. Buckley and F. Iza, Chem. Commun., 2015, 51, 1579. Chem Commun 52:3847-3849. https://doi.org/10.1039/C5CC08289G

21. Nagano H, Shiota G, Arakawa H (2014) Optimization of fluorometric assay for ozone in solution. J Anal Methods Chem 2014:1-5. https://doi.org/10.1155/2014/190937

22. Stratton GW (1985) The influence of solvent type on solvent-pesticide interactions in bioassays. Arch Environ Contam Toxicol 14:651-658. https://doi.org/10.1007/BF01055771

23. Shaw A, Seri P, Borghi CA et al (2015) A reference protocol for comparing the biocidal properties of gas plasma generating devices. J Phys D Appl Phys 48:484001. https://doi.org/10.1088/00223727/48/48/484001

24. Inn ECY, Tanaka Y (1953) Absorption coefficient of ozone in the ultraviolet and visible regions. J Opt Soc Am 43:870-873. https://doi.org/10.1364/JOSA.43.000870

25. Bruck R, Aeed H, Shirin A et al (1999) The hydroxyl radical scavengers dimethylsulfoxide and dimethylthiourea protect rats against thioacetamide-induced fulminant hepatic failure. J Hepatol 31:27-38

26. Wu JJ, Muruganandham M, Chen SH (2007) Degradation of DMSO by ozone-based advanced oxidation processes. J Hazard Mater 149:218-225. https://doi.org/10.1016/j.jhazmat.2007.03.071 